\title{
Coonardoo de Katharine S. Prichard
}

Isabelle Benigno

\section{(2) OpenEdition \\ Journals}

Édition électronique

URL : http://journals.openedition.org/jso/5941

DOI : 10.4000/jso.5941

ISSN : $1760-7256$

Éditeur

Société des océanistes

Édition imprimée

Date de publication : 15 décembre 2009

Pagination : 231-239

ISBN : 978-2-85430-026-0

ISSN : 0300-953x

\section{Référence électronique}

Isabelle Benigno, "Coonardoo de Katharine S. Prichard », Journal de la Société des Océanistes [En ligne], 129 | juillet-décembre 2009, mis en ligne le 30 décembre 2012, consulté le 20 avril 2019. URL : http:// journals.openedition.org/jso/5941; DOI : 10.4000/jso.5941 


\section{Coonardoo de Katharine S. Prichard}

par

\section{Isabelle BENIGNO*}

\section{RÉSUMÉ}

Le roman Coonardoo est l'histoire d'une jeune femme aborigène ainsi nommée qui, dès son enfance, est destinée à s'occuper du ranch de Wytaliba et à prendre soin de son propriétaire, Hugh Watt. Ce dernier étant dans l'impossibilité de reconnaître l'amour qu'il porte à Coonardoo, plonge celle-ci dans un profond désarroi. Il en résulte la destruction de Coonardoo mais aussi celle de Hugh et de toute une communauté. Le cour du livre explore les relations affectives entre les communautés blanche et aborigène et, plus spécifiquement, le racisme comme l'exploitation sexuelle et économique des femmes aborigènes par les hommes blancs. Publié en 1929. le roman choqua les lecteurs de par son évocation de la déchéance des femmes aborigènes mais également de par le choix d'une relation amoureuse entre un Blanc et une Aborigène comme sujet principal du roman. Coonardoo soulève aussi le problème de l'acceptation de l'Australie noire par l' Australie blanche. Pour K. S. Prichard, en 1929, il était évident que l'avenir des deux communautés reposait sur la reconnaissance par l'Australie blanche du lien spirituel unissant les Aborigènes à leur terre.

MoTS-CLÉS: bush, amour interracial, colonisation, dépossession, acculturation

Katharine S. Prichard est née en 1883 à Levuka aux îles Fidji qu'elle quitte avec sa famille pour aller vivre en Tasmanie. Son père est rédacteur en chef d'un journal et sa mère peintre. La famille connaît des difficultés financières importantes qui sont sans doute à l'origine de l'intérêt qu'elle porte aux questions d'injustice sociale et de pauvreté. Il se traduit par un engagement au parti communiste dont la naissance en Australie date de 1920. Cet engagement ne se démentira pas tout au long de sa vie. Elle trouvera dans la

\section{ABSTRACT}

Coonardoo is the story of a young Aboriginal woman who is trained from childhood to be the housekeeper at Wytaliba station and as such is bound to take care of its owner, Hugh Watt. The latter's impossibility to acknowledge his love for Coonardoo, brings terrible misery to her, resulting in the destruction not only of both of them but also of a whole community. At the center of the novel lies the exploration of the emotional lives and the history of interactions between the Black and White communities, more specifically the racism, sexual and economic exploitation of the Aboriginal women by the White men. Published in 1929, the novel shocked the readers not only by its emphasis on the degradation of Aboriginal women but also by the deliberate choice as the main plot of a novel, of the possibility of love between a White man and an Aboriginal woman. Coonardoo also raises the challenging issue of White Australia's acceptance of Black Australia. For K. S. Prichard, in 1929, it was obvious that the future of both communities would be doomed unless White Australia acknowledged Black Australia's spiritual bond with the land.

KeYwords: bush, interracial love, colonization, dispossession, acculturation

philosophie politique de Karl Marx une explication à l'aliénation de l'homme, manipulé par un système recherchant avant tout le profit. La théorie marxiste influencera beaucoup Katharine S. Prichard dans l'écriture de Coonardoo où elle s'attache à décrire un homme prisonnier d'un conditionnement économique et social qui l'empêche de vivre une relation sincère avec la femme qu'il aime.

Une autre influence occupe une place importante dans la construction intellectuelle de

\footnotetext{
* Enseignante, docteur en anglais de l'université Toulouse-le-Mirail, guisa.chassaing@sfr.fr
} 
l'auteur, c'est celle de Carl Gustav Jung (mentionné dans le roman p. 294). Celui-ci considère que l'homme moderne a perdu tout contact avec les mythes des origines et cette perte a gravement endommagé son équilibre psychique. Coonardoo explore l'équilibre psychique précaire de Hugh Watt dont la détérioration est responsable d'un certain nombre de comportements violents. Jung considère que le coupable c'est l'évolution de l'homme tandis que pour Marx, le coupable c'est le système capitaliste. Dans les deux cas, les effets sont désastreux car l'homme se retrouve en position de victime, soumis à des forces qu'il ne parvient pas à contrôler. Il nous paraît important d'avoir à l'esprit ces deux influences pour mieux saisir le positionnement de K. S. Prichard dans ce roman.

Outre l'environnement intellectuel, il convient également de replacer l'œuvre dans le contexte économique, politique et social australien à l'époque où le roman est rédigé. Coonardoo est publié en 1929, en pleine Dépression, dans un contexte économique qui fragilise encore un peu plus la minorité aborigène. Comme le souligne l'historien Henry Rowley :

"One of the effects of the Great Depression, all over Australia, seems to have been a more rigid containment in institutions, where conditions were probably worse than ever before, with enduring effects on Aboriginal attitudes. » (Rowley, 1972 : 281)

Le contexte politique est marqué par une bouffée de violence en Australie occidentale : une tribu aborigène y est complètement décimée en réponse à une " provocation ». Il n'en faut pas davantage pour que les esprits s'échauffent et que non content de massacrer toute une tribu, on tue son chef en pleine ville de Perth. Cet épisode sanglant, coïncidant avec la publication de $\mathrm{CoO}$ nardoo, est l'un des derniers soubresauts de la colonisation.

Dans ce contexte tendu, le roman de Katharine S. Prichard ne joue pas l'apaisement : il se veut en effet, une dénonciation des conséquences néfastes de la colonisation sur les populations aborigènes doublement soumises. D'une part, elles sont économiquement dépendantes des grands propriétaires fonciers blancs; d'autre part, depuis le milieu du $\mathrm{XIX}^{\mathrm{e}}$ siècle, des missions ont été créées et placées sous le contrôle de missionnaires. Leur but est double : convertir les Aborigènes à la religion chrétienne mais également aux us et coutumes de l'homme blanc. Toutefois, ne parvenant pas à anéantir totalement la culture aborigène, le nouvel État, né de la fédération des colonies en 1901, se dote d'une constitution où le principe d'exclusion est claire- ment affiché. Les Aborigènes y sont considérés comme des « citoyens de seconde zone » qui ne sauraient bénéficier des mêmes droits que les citoyens blancs. Cette politique d'exclusion raciale vise une homogénéisation de la population par le biais notamment de déplacements forcés des communautés aborigènes vers des réserves. L'une des mesures les plus destructrices consiste à enlever de force des milliers d'enfants aborigènes de sang-mêlé à leurs parents pour les «blanchir». Cette déportation systématique part du postulat qu'il faut «assimiler la race ». La politique d'assimilation est cependant de plus en plus contestée par les aborigènes qui refusent d'être représentés comme de simples victimes consentantes. Les décennies 1920-1930 sont marquées par une mobilisation des Aborigènes au sein de groupements politiques dont la naissance remonte au milieu des années 1920. L'un des premiers mouvements de protestation aborigène créés est La Ligue pour le Progrès et L'avancement des Aborigènes dont le but est de dénoncer les préjudices subis par ces derniers mais également de s'opposer et résister aux politiques de ségrégation mises en place par les autorités australiennes.

Avec la publication de ce roman, Katharine S. Prichard confirmait son statut d'écrivain. En 1916, son premier roman The Pioneers, une histoire de vol de bétail sur fond de romance dans le Gippsland, lui avait valu de remporter le premier prix du Hodder and Stoughton All Empire Novel Competition, un concours destiné à révéler de nouveaux talents. Au début des années 1920, elle s'établissait avec sa famille en Australie occidentale et entreprenait d'écrire une série de romans ayant pour toile de fond les conditions de vie rudes auxquelles étaient confrontés ses habitants. En 1926, paraissait Working Bullocks, un roman dans lequel elle s'intéressait à une communauté de bûcherons et à l'influence qu'exerçait sur cette dernière un militant communiste.

Son intérêt pour les gens ne se démentit pas dans Coonardoo. Lors de sa parution, le roman suscita la controverse. Beaucoup de critiques s'indignèrent $\mathrm{du}$ fait qu'une femme écrivain blanche ait pu choisir comme thème central de son roman l'amour interracial. Bien que nul n'ignorât que cela existait, il n'en demeurait pas moins que le sujet était tabou. Katharine S. Prichard dénonçait l'hypocrisie de ses congénères espérant ainsi éveiller les consciences. À travers cette histoire, elle nous livrait un témoignage poignant sur une minorité dépossédée dont le mode de vie avait été irrémédiablement bouleversé par la colonisation et ses méfaits. 
Roman éponyme, Coonardoo peut en effet se lire comme le récit d'une triple destruction : destruction d'une femme; destruction d'un domaine ; destruction de l'identité aborigène.

\section{La destruction d'une femme}

Coonardoo est une enfant de la tribu des « Noueux » qui vit sur le domaine de Wytaliba que dirige $\mathbf{M}^{\mathrm{me}}$ Bessie Watt depuis le décès de son mari. Son fils, le jeune Hugh, a grandi aux côtés de Coonardoo. Le roman s'ouvre sur le départ de Hugh en pension et la nostalgie passagère que ressent Coonardoo, toute la scène étant en focalisation interne. Coonardoo est omniprésente dans la narration de façon directe (début et fin de roman) ou indirecte; lorsque Hugh la chasse, elle demeure présente à travers la chanson aborigène traditionnelle que fredonne son fils :

« Ma mère, je suis là à pleurer sur ton sort, mais je reviendrai avec des choses à manger qui te feront plaisir. » (Prichard, 1991 : 284)

De même, des bribes de nouvelles parviennent à la station qui permettent de se représenter sa situation et plus généralement celle des femmes aborigènes qui font l'objet de mauvais traitements (exploitation sexuelle). Ainsi, Sam Geary apostrophe-t-il Hugh dans un bar à propos de Coonardoo :

«Dis-donc, Youie! Beugla-t-il, devine un peu qui j'ai vu au port l'autre jour? Coonardoo ! Et jamais on n'a vu de vieille ruine pareille ! Je ne l'aurais jamais reconnue si elle ne s'était pas mise à piailler [...] sur le point d'être internée dans l'île. » (Prichard, 1991 : 292)

La scène finale est également perçue à travers le regard de Coonardoo, ce qui permet au lecteur de ressentir une profonde empathie pour le personnage mais également de prendre la mesure de sa détresse physique et morale. La fin offre aussi un contraste saisissant avec les premières lignes $\mathrm{du}$ roman dont l'atmosphère est empreinte d'un bonheur teinté de nostalgie :

« Coonardoo chantait. [...] S'élançant, retombant, les mots s'entrechoquaient et s'envolaient mystérieusement. » (Prichard, $1991: 15$ )

La chanson du bonheur devient un chant funèbre à la fin du roman :

« Elle fredonna un moment et s'allongea. Ses bras, ses jambes s'écartèrent; on aurait dit des bouts de bois noircis et brisés, à côté du feu. » (Prichard, 1991 : 305)
Entre ces deux moments, il y a l'espace de la narration dont le fil conducteur n'est autre que la destruction de Coonardoo, processus où causes sociales et psychologiques sont étroitement liées : mainmise des Blancs sur la terre et recours à une main-d'œuvre aborigène non-payée d'une part; pratique fort répandue parmi les Blancs de considérer les femmes aborigènes comme leur propriété sexuelle d'autre part. Deux hommes jouent un rôle prépondérant dans la destruction de Coonardoo : Sam Geary et Hugh Watt.

Hugh est décrit dans le roman comme beaucoup moins dangereux que Geary dont le comportement envers les femmes est qualifié de prédateur. Les sentiments de Hugh vis-à-vis de Coonardoo sont excessivement ambigus : une très grande complicité l'unit à Coonardoo depuis son enfance mais il demeure prisonnier des préjugés de l'homme blanc. Ainsi après le décès de Warieda (le mari de Coonardoo), Hugh considère Coonardoo comme sa "propriété » bien qu'il ne soit nullement disposé à offrir son amour pour des raisons morales. Hugh prend peu en considération les sentiments de Coonardoo car cela supposerait qu'il la traite en égale, ce qui est impensable compte tenu du contexte historique et social. Coonardoo ne comprend pas non plus les motivations de Hugh, notamment lorsque ce dernier la rejette sexuellement alors que dans le même temps il en revendique la " possession ». Aux yeux de l'auteur, Hugh est le digne représentant de la classe des possédants qui soumet toute chose à sa volonté. Hugh et Coonardoo sont aux antipodes et pourtant ces antipodes se rejoignent une seule fois dans le roman dans des circonstances particulières pour Hugh : celui-ci, miné par la solitude et la maladie après le décès de sa mère, cède à Coonardoo. $\mathrm{Ce}$ sera la seule et unique fois et il n'en sera plus jamais question :

«Hugh la prit dans ses bras et s'abandonna à l'esprit qui, de bien loin semblait-il, l'attirait vers la source commune où sa vie se mêlait à celle de Coonardoo. » (Prichard, 1991 : 98)

Il s'agit d'un épisode central du roman car il révèle ce que Hugh a tenté jusque-là de laisser enfoui au plus profond de lui-même (les psychanalystes parlent de refoulement), à savoir cette affinité profonde qu'il ressent à l'égard des aborigènes. Les barrières mentales mises en place pour maintenir à distance ses sentiments cèdent car il n'est plus physiquement en état de résister. Ce moment-clé lui permet de connaître une harmonie du corps et de l'esprit. Mais le répit est de courte durée. Dès qu'il recouvre toute sa lucidité, il redevient cet homme pétri de préjugés, en proie 
à un dilemme qu'il est incapable de résoudre et dont Coonardoo fait les frais.

Cet épisode peut se lire également comme une illustration des théories de Carl Gustav Jung dont nous avons souligné l'influence dans l'introduction. Hugh confronté à une crise majeure après le décès de sa mère est en définitive incapable de transférer l'amour qu'il lui porte vers une autre femme. Son admiration pour sa mère est sans bornes, elle est une sorte de déesse qu'il vénère et pour laquelle il est prêt à tout sacrifier. Il s'interdit d'aimer Coonardoo et d'en faire sa femme en dépit de l'attirance profonde qu'il ressent, préférant endosser l'habit de protecteur, prenant fait et cause pour elle, jusqu'au moment où elle détruit son fantasme. C'est Phyllis, l'une des filles de Hugh qui se livre à cette analyse à la fin du roman :

«Tu n’as jamais entendu parler d'un type nommé Jung? [...] Tu sais, Bill, j'ai la conviction que notre Youie a pris ma mère comme la plupart des hommes prennent une gin et que Coonardoo, elle, a été une sorte de fantasme pour lui. » (Prichard, 1991 : 294295)

Coonardoo détruit le fantasme de Hugh en se donnant à Sam Geary qui fait précisément ce que Hugh s'interdit de faire. La révélation de cette relation déclenche une scène de jalousie d'une rare violence, scène au cours de laquelle Hugh ne prend en considération ni ses propres défaillances ni le dévouement sans faille de Coonardoo. Pour Katharine S. Prichard, Hugh est trop imbu de son statut économique mais également de son sentiment de supériorité (supériorité de la culture et des valeurs morales de l'homme blanc). Cela a pour conséquence le bannissement de Coonardoo et sa lente déchéance : son exil la conduit notamment à bord d'un perlier où Coonardoo n'est que l'une des nombreuses victimes de l'exploitation sexuelle par les Blancs et dont Sam Geary est sans conteste le meilleur représentant dans le roman.

À la différence de Hugh dont les bonnes intentions à l'égard des Aborigènes révèlent au bout du compte une personnalité pétrie de contradictions, Sam Geary incarne le prototype du pionnier déterminé à survivre dans cet environnement hostile et à réussir quel qu'en soit le prix. Il ne cache pas son intérêt pour la gent féminine et entretient des relations sexuelles avec des femmes aborigènes, ne s'embarrassant d'aucune considération morale. Il n'est guère étonnant que Geary s'intéresse à Coonardoo et profite de la première occasion où il se retrouve seul avec cette dernière. Geary est tenu pour responsable de la destruction de Coonardoo mais aux yeux de l'auteur, la plus grande part de responsabilité incombe à Hugh.

Tout le roman s'attache à démontrer les qualités exceptionnelles de cette femme, prisonnière des désirs des hommes. Coonardoo doit son nom à un puits, élément essentiel à la survie d'une station. Lorsque Hugh ne l'accepte pas en tant que partenaire sexuelle à part entière, Coonardoo éprouve un profond sentiment de frustration : sa relation « stérile » à Hugh fait écho à la stérilité de la terre soumise à des périodes de sécheresse intense. On peut également lire l'épisode où Coonardoo cède à Geary comme l'expression d'une volonté de mettre fin à cette période de stérilité :

"Sa stérilité avait fait d'elle une morte-vivante. L'étreinte de Geary avait libéré un instinct qu'on aurait dit inflammable, qui avait prise sur elle et qui essaimait. » (Prichard, 1991 : 266-267)

Dernier épisode mettant en évidence ce jeu sur la symbolique de la fertilité/stérilité, celui du bannissement de Coonardoo de Wytaliba: il coïncide avec une période de sécheresse extrême (sécheresse intime). L'un des membres de la tribu des " Noueux », associe la stérilité de la terre au départ de Coonardoo. Même le puits s'assèche peu à peu. La source de joie, de fertilité et de croissance que représentait Coonardoo s'est définitivement tarie. Hugh sombre dans un état de semi-démence tandis que Wytaliba s'enfonce dans une situation de plus en plus délicate. $\mathrm{La}$ destruction de Coonardoo est directement responsable de la destruction de Wytaliba.

\section{La destruction de Wytaliba ou la dure réalité du bush}

Le roman de Prichard se veut un portrait fidèle de la vie dans les stations (isolement ; conditions climatiques extrêmes), de sa dureté et de la nécessaire mais difficile adaptation de l'homme blanc à cet environnement dont il ignore tout. Deux points de vue s'opposent dans le roman. Celui de $\mathbf{M}^{\text {me }}$ Bessie, Hugh, Phyllis, Coonardoo, des hommes et des femmes profondément attachés à Wytaliba et sa terre; celui de Jessica et Mollie d'autre part, qui détestent le bush et se saisissent du premier prétexte pour le fuir. Les difficultés auxquelles sont confrontés les propriétaires d'exploitations sont évoquées, qu'il s'agisse des longues périodes de sécheresse et de leurs conséquences économiques (pressions financières exercées par les banques) mais également de la solitude sexuelle qui amène les hommes à cher- 
cher des partenaires parmi les femmes aborigènes. Pour rompre cette solitude, Sam Geary invite Hugh à ne pas se montrer trop exigeant avec lui-même sous peine de sombrer. C'est précisément ce qui arrive à Hugh. Geary est incontestablement prédateur mais c'est aussi un survivant, capable de s'adapter à toutes les situations et d'en tirer profit, au sens propre du terme puisque à la fin du roman, grâce aux difficultés financières de Hugh, Geary s'empare de Wytaliba. Hugh aura finalement tout perdu : Coonardoo et la station pour laquelle sa mère s'était battue.

Vivre dans le bush est en effet un combat permanent. Prichard s'en fait l'écho à travers la description minutieuse de Wytaliba où Blancs et Aborigènes travaillent ensemble dans une relation de respect mutuel jusqu'au bannissement de Coonardoo. À partir de ce moment, les choses se retournent contre Hugh : la pression des banques, la sécheresse mais aussi et surtout le fait qu'il s'aliène par son comportement, le soutien de la tribu des « Noueux ». Ce qui est mis en évidence c'est la relation d'interdépendance entre Blancs et Aborigènes. Sans la maind'œuvre aborigène rétribuée en nature, il est évident que Wytaliba ne peut survivre. Le point de vue de Prichard est clair: l'exploitation de la terre a été rendue possible par l'exploitation de la main-d'œuvre aborigène.

L'auteur n'est pas seulement sensible aux enjeux économiques. Elle se montre aussi fine observatrice de l'environnement naturel dont la beauté est évoquée à de multiples reprises: faune, flore, changements de saisons sont minutieusement restitués laissant percevoir un attachement sincère pour ces grands espaces :

«Les troncs des eucalyptus blancs comme de la craie, bordaient le lit asséché du cours d'eau sur toute sa longueur. Plus loin surgissait, dénudée et rouge vif, la ligne en dents de scie des collines, dont la partie basse avait pris la nuance fauve du spinifex [...]. Loin, toujours plus loin, au fil des méandres d'une interminable piste qui franchissait la plaine, puis les étendues de terres, où la mulga se dresse, rigide et brillante comme du métal, depuis le temps qu'elle est morte et que le soleil l'a décolorée. » (Prichard, 1991 : 16)

Cette nature est exigeante et l'on sent poindre une certaine forme de mépris pour des personnages comme Jessica et surtout Mollie qui ne sont pas à la hauteur du défi qui s'offre à elles. La première préfère retourner en ville après quelques mois passés à Wytaliba tandis que la seconde, bien que mariée à Hugh, ne partage aucune des vues de ce dernier, qu'il s'agisse du travail sur la propriété ou des relations avec les
Aborigènes. Hugh consacre toute son énergie à Wytaliba : il a donc besoin d'une femme capable de tenir un intérieur et de diriger les domestiques comme sa mère le faisait. Mollie prend son rôle de maîtresse de maison très à cœur dans un premier temps. Toutefois, elle entend exercer ce rôle sans partage. Bonne à tout faire dans une pension de famille avant son mariage, elle s'imagine tenir enfin une revanche sur une existence étriquée et fade :

«Imaginez, avoir toutes ces servantes ! Transportée à l'idée de sa grandeur, Mollie houspilla les gins comme $\mathbf{M}^{\mathrm{me}}$ Armstrong l'avait houspillée si souvent elle-même. » (Prichard, 1991 : 134)

Le narrateur/auteur a peu d'affection pour Mollie dont le comportement nous est présenté comme inadapté à la situation. Loin de se faire respecter par les domestiques, son autoritarisme est tourné en dérision. Elle n'a ni l'étoffe ni le charisme de $\mathbf{M}^{\text {me }}$ Bessie Watt. Mollie ne se sent exister qu'au travers de ce qu'elle possède :

«Pour la première fois de sa vie, Mollie avait le sentiment d'être propriétaire. Être propriétaire de cette cuisine, de ces pots et de ces casseroles était une sensation nouvelle. » (Prichard, 1991 : 134)

Mollie confond être et avoir, confusion qui du point de vue du narrateur/auteur, est une des principales caractéristiques morales des Blancs. À trop vouloir posséder, Mollie finit par ne plus rien contrôler. Elle ne parvient d'ailleurs pas à s'imposer comme l'épouse de Hugh. L'ombre de Coonardoo est omniprésente, ce qu'elle vit très mal. Épuisée après cinq grossesses (toutes donnent naissance à des filles comme s'il y avait chez Hugh une forme d'impuissance), Mollie décide de partir en ville où elle compte sur les largesses financières de Hugh pour mener grand train.

Le portrait de Mollie est sans concession : la vie dans le bush ne tolère aucune forme de faiblesse morale ou physique :

« Mais ici, dans un pays aux horizons sans fin, sous une voûte céleste illimitée, vivre replié sur soi-même revenait à se décomposer de l'intérieur. Pour survivre, il fallait rester porté par le courant de vie qui émanait de cette terre. Il fallait en faire partie et l'accompagner, pour pouvoir travailler, pour évoluer avec lui. » (Prichard, $1991: 163$ )

Mollie a l'impression d'être déplacée dans cet environnement rude et hostile où toute manifestation de sensibilité est à proscrire. Si la mère de Hugh était aussi à l'aise à Wytaliba, c'est parce qu'elle était dotée des qualités utiles à la survie dans le bush, à savoir énergie et résistance. Comme le souligne fort justement Xavier Pons : 
«Comme la plupart des sociétés occidentales, l'Australie est dominée par le "pouvoir masculin" qui y prend parfois des formes assez outrancières. Cela s'explique par l'histoire du pays. Qu'il s'agisse de l'univers pénitentiaire ou de celui des pionniers qui exploraient et défrichaient le bush, le berceau de la civilisation australienne fut une rude école, où la force physique, l'énergie et la résistance constituaient les qualités les plus utiles à l'individu et où, par contraste, la délicatesse et la sensibilité n'avaient guère de place. » (Pons, 1983 : 158)

L'admiration que porte l'auteur à cet environnement naturel et à ceux qui lui sacrifient tout, l'amène à lui accorder dans le roman une place plus importante que certains personnages, ce qui aux yeux de certains critiques constitue une faiblesse dans l'économie générale du récit. Le bush est en effet présenté comme le théâtre d'enjeux économiques ayant de fortes répercussions sociales, morales et donc nécessairement identitaires sur les communautés aborigènes.

\section{La destruction de l'identité aborigène}

Celle-ci découle de trois facteurs étroitement liés : la possession de la terre par les Blancs; l'exploitation sexuelle des femmes aborigènes; l'interférence des Blancs dans la vie sociale et spirituelle des aborigènes.

L'identité morale des Blancs est liée à leur pouvoir et statut économique. Alors qu'il n'est qu'un enfant, Hugh nous est dépeint sous les traits d'un garçon autoritaire tandis que Coonardoo se comporte en petite fille obéissante. Les valeurs morales de la culture blanche sont dominantes et il ne viendrait à l'esprit de personne de contester cet état de fait. Au début du roman, nous apprenons que la mère de Coonardoo, Maria, est morte sous les coups de Ted Watt, le père de Hugh :

« Ted avait tué le chien de Maria [...]. Elle lui aurait répondu refusant de faire ce qu'il lui disait un jour qu'il était soûl. Il l'avait jetée en bas de la vérandah à coups de botte. Quelques jours après, Maria rendait l'âme. " (Prichard, 1991 : 24)

La violence physique de Ted Watt n'est pas de nature intrinsèque. C'est l'alcool qui en est à l'origine :

«De l'avis unanime, Ted Watt n'était pas pire que les autres, sauf quand il avait bu. Non, il ne supportait pas l'alcool, quelques verres suffisaient à le rendre fou furieux. » (Prichard, 1991 : 24)

Le crime de Ted Watt ne restera cependant pas impuni puisqu'il trouvera la mort un mois plus tard lors d'une beuverie. De même, Hugh Watt, son fils, verra-t-il Wytaliba tomber aux mains du cupide Sam Geary, se retrouvant ainsi sans le sou après qu'il eut banni Coonardoo. Cette dernière se voit ainsi « vengée » alors même que l'idée de se venger d'une quelconque manière lui est totalement étrangère. Après la mort de Maria, elle n'éprouve aucun ressentiment vis-à-vis de Ted Watt. Les rapports que Coonardoo entretient avec Hugh sont quant à eux basés sur des liens tissés tout au long de l'enfance. Au début du roman, elle est dans la souffrance de la séparation :

« Ne partait-il pas demain ? Pour aller en pension? Et ils ne joueraient plus ensemble dans le jardin là-bas près de l'éolienne. » (Prichard, 1991: 7)

Plus tard, lorsque Hugh est terrassé par la maladie, elle montre un dévouement sans faille, attentive à ses moindres désirs.

Hugh, en revanche, ne partage pas le même sens du «dévouement ». Bien que Coonardoo ait été sa compagne de jeu, puis son amante, il n'hésite pas à se comporter de manière tout aussi brutale et inhumaine que son père vis-à-vis de Maria, ignorant totalement le point de vue de Coonardoo :

«Les paroles les plus ignobles, un torrent de mots durs et suprêmement cruels jaillirent de la bouche de Hugh, debout près du feu. Il frappa Coonardoo au visage lorsqu'elle releva la tête pour le regarder en pleurant et l'implorer sans un mot. » (Prichard, 1991 : 276-277)

Les comportements violents du père et du fils sont emblématiques des multiples pratiques discriminatoires dont les aborigènes font l'objet. À plusieurs reprises, le roman se fait l'écho de ces dernières qu'il s'agisse d'expéditions punitives menées par la police ou de la pratique du blackbirding :

« On ramenait les nègres enchaînés, une lanière de cuir autour du cou nouée aux étriers. Vingt ou trente à la fois [...]. Je connaissais un perlier qui pratiquait le blackbirding; son équipage de garçons ramassés à Swan Point est devenu si dangereux qu'il a dû les expédier par-dessus bord en pleine mer. » (Prichard, $1991: 156)$

Si ces comportements sont admis, c'est en raison de l'absence de statut légal ou économique des Aborigènes. Ces derniers coopèrent de manière volontaire aux travaux de l'exploitation en échange de nourriture, de vêtements et de quelques cadeaux parfois, entretenant une dépendance des Aborigènes vis-à-vis de leurs employeurs blancs. Cette relation de dépendance 
est présentée comme malsaine dans le roman car elle entraîne une baisse sensible des pratiques de chasse et de cueillette des Aborigènes ainsi qu'une sédentarisation forcée. Or, il faut le rappeler, les Aborigènes entretiennent avec la terre un lien spirituel très fort. Le paysage contient la trace des événements du Temps du Rêve. Ce n'est pas une étendue géographique quelconque. Il porte les empreintes du passage des héros du Temps du Rêve dans ses arbres, ses sources, ses rochers. La pensée aborigène est une pensée totémique. Pour que le lien entre l'homme et la terre soit préservé et entretenu, chaque Aborigène en tant que descendant d'un Ancêtre, doit célébrer un certain nombre de rites qui l'amènent à parcourir de grandes distances. En les forçant à se sédentariser, les Blancs ont complètement bouleversé ce rapport à la Terre-Mère tant d'un point de vue matériel que spirituel. Les bonnes intentions des Blancs dont l'auteur se fait l'écho, ne sauraient compenser les souffrances dans lesquelles les Aborigènes ont été plongés suite à l'appropriation de leurs terres. Dans le roman, les rapports entre les deux communautés sont placés sous le signe de la bienveillance. $\mathrm{M}^{\mathrm{me}}$ Bessie est un autocrate octroyant aux Aborigènes une marge d'indépendance importante en ce qui concerne leur organisation sociale et la conduite de leur vie spirituelle.

Il est toutefois un domaine où des différences très nettes s'expriment, c'est celui de la sexualité. Autant $\mathrm{M}^{\mathrm{me}}$ Bessie exprime son admiration pour des pratiques ancestrales telle que le corroboree:

« Avec grâce et agilité, sautant d'un pied sur l'autre, retombant à pieds joints, genoux écartés, il avait mimé le jeune homme "chantant toujours" en train de danser son corroboree, audacieux et enjoué. » (Prichard, $1991: 37)$

Autant elle se montre très critique à l'égard de certains rites initiatiques qu'elle considère comme immoraux et sadiques :

« $\mathrm{M}^{\mathrm{me}}$ Bessie avait parfois des accès de répulsion envers les Noirs [...]. Des pratiques qu'elle jugeait immorales l'avaient dégoûtée. » (Prichard, 1991 : 40)

Son attitude, mélange de fascination et de répulsion, est caractéristique de la conception que les Blancs se font des Aborigènes à l'époque où le roman est écrit : ces derniers sont considérés comme des êtres primitifs qu'il convient d'éduquer. Ce sont des enfants qui n'ont aucune conscience du bien et du mal :

«Quand les Blancs ne leur ont pas expliqué, les Noirs ne voient pas le lien entre la naissance des enfants et un rapport sexuel fortuit. » (Prichard, 1991 : 41)
C'est parce qu'elle considère qu'elle a un devoir d'éducation morale que $\mathrm{M}^{\mathrm{me}}$ Bessie intervient dans les arrangements matrimoniaux concernant Coonardoo et Warieda. Mais cette intervention a des conséquences fâcheuses : elle déclenche un affrontement entre Warieda et un Aborigène d'une autre tribu désireux de s'emparer de Coonardoo tandis que dans le même temps Geary qui convoite également Coonardoo, tente d'amadouer son père avant d'essayer de l'enlever.

L'intervention des Blancs dans l'organisation sociale interne à la tribu est présentée comme inappropriée et dommageable pour l'équilibre social. $\mathrm{M}^{\mathrm{me}}$ Bessie prend conscience que ce qui est en jeu à travers le cas particulier de Coonardoo, s'apparente à une lutte d'influence destinée à asseoir les valeurs morales véhiculées par la culture des Blancs au détriment de la culture aborigène :

« Non pas que $\mathrm{M}^{\mathrm{me}}$ Bessie cherchât à l'arracher à son élément. Pas du tout, mais elle redoutait que la petite ne fût soumise à une influence plus forte que la sienne. » (Prichard, 1991 : 45)

Cependant, il est intéressant de noter qu'il n'y a pas uniformité des points de vue et des comportements concernant l'attitude des Blancs visà-vis des Aborigènes, notamment en matière de moralité sexuelle. L'attitude de Sam Geary est jugée profondément amorale par l'auteur mais elle s'explique par un instinct de survie ainsi que par un besoin évident de satisfaction sexuelle qu'il semble assumer et même revendiquer. Il n'hésite d'ailleurs pas à se faire le chantre de la polygamie, citant même la Bible :

«Sam était debout près du bar, un énorme feutre neuf sur la tête, le visage en feu, congestionné, l'air à vif. "Mais le roi Salomon aimait de nombreuses et étranges femmes en même temps que la fille de Pharaon" Hugh l'entendit déclamer comme bien des fois déjà. » (Prichard, 1991 : 291-292)

Ce que Sam Geary se garde bien de mentionner au passage c'est le résultat de ces relations sexuelles entre Blancs et femmes aborigènes, à savoir de nombreux enfants métis. La question du métissage est abordée dans le roman à travers deux exemples: d'une part celui de Geary et sa nombreuse progéniture dont il se désintéresse complètement; d'autre part, celui de Winni, l'enfant de Hugh et Coonardoo. Cet enfant est immédiatement reconnu par Hugh comme étant le sien :

«Debout près de Coonardoo, Hugh regardait l'enfant. Il savait que c'était son fils. » (Prichard, 1991 : 120) 
Hugh entretient avec Winni une relation de complicité tout en évitant de lui révéler la vérité sur ses origines. Pourquoi ? Hugh s'interdit-il de reconnaître cet enfant par confort moral ou bien par respect pour les Aborigènes et ce afin de ne pas couper Winni de ses racines? C'est la deuxième option qui est privilégiée par Hugh :

« Son amour et sa fierté à lui étaient-ils plus grands que la fierté et l'amour de Warieda? Se demandait Hugh. Il avait de l'affection pour le petit, mais pouvait-il apporter à Winni ce que lui apportait Warieda? Lui apprendre à faire ce qu'il voulait des chevaux, le préparer à une vie indépendante dans son environnement naturel ?» (Prichard, $1991: 182)$

Son attachement à Winni le conduit à le préférer à ses propres enfants et sa femme. Son mariage avec Mollie est postérieur à sa relation avec Coonardoo et la naissance de Winni :

«Coonardoo leva les yeux vers Hugh puis les ramena vers le petit. Mais Hugh avait tout lu dans son regard : elle l'avait attendu, elle ne demandait rien, n'espérait rien; elle était prête à servir son épouse, cette femme blanche qu'il avait ramenée pour partager sa vie. » (Prichard, 1991 : 120)

Le dévouement de Coonardoo ne sera jamais payé de retour. Jalouse de la complicité unissant Hugh à celle-ci, Mollie n'a de cesse de dénigrer Coonardoo et son «morveux de métis ». Hugh condamne l'attitude de sa femme, qui déshonorée par la présence de l'enfant de Hugh et Coonardoo, souhaite que son époux les chasse purement et simplement. À ce stade de la narration, un tel geste est impensable pour Hugh :

« Il réagissait aux choses comme Warieda et Coonardoo. À ses yeux, exiger de les en chasser comme le faisait sa femme, était indéfendable; ça ne se faisait pas. Il ne fallait pas le faire ; il ne le ferait pas. » (Prichard, 1991 : 189)

Cette prise de position prend un parfum particulier au regard du comportement de Hugh vis-à vis de Coonardoo. À partir de ce moment, un fossé se creuse entre Hugh et Winni, fossé qui ne sera jamais comblé. Winni n'est d'ailleurs pas jugé « digne » d'hériter de Wytaliba et de faire fructifier l'exploitation.

\section{Conclusion}

En choisissant comme thème central du roman l'amour interracial, l'auteur a pleinement conscience que la problématique du métissage est cruciale dans toute approche des relations entre Blancs et Aborigènes et, a fortiori, dans toute approche de l'identité aborigène. Cette problématique a fait et fait débat notamment chez nombre d'intellectuels aborigènes. D'aucuns considèrent que l'on est aborigène ou que l'on ne l'est pas indépendamment du degré de métissage. C'est la position que revendique un écrivain comme Kim Scott : l'aboriginalité est une notion culturelle et non une donnée génétique. D'autres écrivains ne partagent pas ce point de vue, arguant que certaines personnes se revendiquent aborigènes pour en retirer des avantages qui sont au demeurant fort limités (on a assisté dans les années 1990 à l'éclosion d'un certain nombre de cas d'impostures, le plus célèbre étant celui de Leon Carmen publiant sous le nom de Wanda Koolmatrie). Le grand mérite de Katharine S. Prichard réside incontestablement dans la désignation comme personnage principal du roman d'une Aborigène, ce qui au regard du contexte politique constitue une démarche courageuse. Toutefois, Coonardoo demeure prisonnière du désir et des sentiments de l'homme blanc. Comme l'ont souligné de nombreuses critiques, Coonardoo est un roman mettant l'accent sur «la subjectivité de l'homme blanc». Le roman pose un certain nombre de questions qui n'avaient pas été abordées jusque-là mais les réponses apportées restent celles d'un écrivain blanc : on peut d'ailleurs faire le même reproche à un autre roman publié en 1937 par un écrivain blanc également, Capricornia, dans lequel l'auteur va plus loin que Katharine S. Prichard sur la question du métissage mais avec des prises de position sur la politique d'assimilation très contestables pour le lecteur d'aujourd'hui. En outre, on peut regretter un certain penchant manichéiste, notamment en ce qui concerne l'opposition des deux logiques économiques: celle du capitalisme foncier blanc présenté comme destructeur des valeurs sociales et spirituelles dont la société aborigène est porteuse.

L'interrogation majeure, sous-jacente au roman, savoir comment réconcilier les deux communautés, ne trouve pas de réponse satisfaisante. Parler de réconciliation au début des années 1930 était, pour le moins, prématuré, les esprits n'y étant guère préparés. Avant d'envisager la mise en œuvre d'un tel processus, il était nécessaire de dresser un état des lieux. Le roman de Katharine S.Prichard met l'accent sur les injustices commises par les Blancs envers les Aborigènes qui ont été spoliés de leurs terres et dépossédés de leur culture.

Le processus politique conduisant à la restitution de leurs terres aux Aborigènes ne s'est véritablement enclenché qu'au milieu des années 1970 avec The Aboriginal Land Rights Act (1976) 
suivi en 1980 par la création des Conseils de la Terre aborigène, deux initiatives fortes prises durant la période où les travaillistes étaient au pouvoir. Le préjudice moral subi par les Aborigènes est, quant à lui, beaucoup plus difficile à mesurer et à réparer car cela suppose, du point de vue de l'auteur, une remise à plat de l'éthique capitaliste. Comme nous l'avons précédemment souligné, Coonardoo fut publié en 1929 en pleine déroute du système capitaliste. Il est donc logique que l'auteur ait cherché d'autres alternatives à un système qui de plus allait à l'encontre de ses convictions personnelles. Ce faisant, elle invite ses lecteurs à puiser dans les valeurs culturelles aborigènes (attachement à la terre et prééminence des liens communautaires sur l'individu) afin de se ressourcer moralement et de construire un avenir où Blancs et Aborigènes seraient réconciliés. Une telle démarche peut paraître quelque peu utopiste au regard du contexte politique.

Néanmoins, il est indéniable que les idées portées par le roman de Katharine S. Prichard ont permis à la communauté blanche australienne de prendre progressivement conscience des injustices commises par le passé et de la nécessité de revenir aux valeurs essentielles contenues dans la culture aborigène comme moteur de construction identitaire :
« Those who wish to present a critique of individualism point out that Aboriginality is about community; those who wish to highlight the detrimental effects of industrialisation on the environment point to the Indigenous people as the original conservationists. We present a remaining though strategically distant, image of what has been lost, and what could be regained. " (Dodson, $2003: 36)$

\section{BIBLIOGRAPHIE}

BENIGNO Isabelle, 2006. Évolution des représentations de l'Aboriginalité dans la littérature australienne du $\mathrm{xx}^{\mathrm{e}}$ siècle, thèse de doctorat d'anglais, Université de Toulouse Le Mirail, sous la direction de M. Xavier Pons.

Dodson Michael, 2003. The end in the beginning: re(de)fining Aboriginality, Blacklines, Contemporary Critical Writing by Indigenous Australians, Melbourne, Melbourne University Press.

Pons Xavier, 1983. L'Australie et ses populations, Paris, éditions Complexe.

Prichard Katharine Susannah, 1991. Coonardoo, traduction de Jean-Paul Delamotte et Hélène Jacomard, Paris, La Petite Maison.

-, 2000. Coonardoo, Sydney, Angus and Robertson.

Rowley Henry, 1972. The Destruction of Aboriginal Society, Harmondsworth, Penguin. 
